\title{
A concise route to phytosphingosine from lyxose
}

\author{
Chun-Cheng Lin,,* Gang-Ting Fan ${ }^{\mathrm{a}, \mathrm{b}}$ and Jim-Min Fang \\ a Institute of Chemistry, Academia Sinica, Nankang, Taipei 115, Taiwan \\ ${ }^{\mathrm{b}}$ Department of Chemistry, National Taiwan University, Taipei 106, Taiwan
}

Received 18 December 2002; revised 5 May 2003; accepted 15 May 2003

\begin{abstract}
Phytosphingosine was synthesized from the commercially available D-2,3- $O$-isopropylidene-D-lyxofuranose in $28 \%$ overall yield by a six-step procedure. This procedure is expedient and flexible for introduction of other lipid moieties on the phytosphingosine structure to make a variety of derivatives that can support the further exploration of their related biological functions. (C) 2003 Published by Elsevier Science Ltd.
\end{abstract}

The biomembrane components sphingophospholipids and glycosphingolipids are of physiologically importance in cell proliferation, differentiation, adhesion, neuronal repair, and signal transduction. ${ }^{1-3}$ A sphingolipid consists of an hydrophilic head (e.g. saccharide or phosphate) and a lipophilic tail (e.g. ceramide). $\alpha$-Galactosyl ceramide ( $\alpha$-GalCer), a glycolipid originally extracted from marine sponge Agelas mauritinu, ${ }^{4}$ has been recently shown to recognize and bind with CD1d molecules on an antigen-presenting cell surface. ${ }^{5}$ Such an interaction presents the sugar moiety of the antigen to a receptor on natural killer T-cells (NKT cells) to activate the immune system. ${ }^{6}$ The structure of $\alpha$-GalCer incorporates a phytosphingosine (1) type ceramide and galactose with $\alpha$ glycosidic linkage.

Many methods for the synthesis of phytosphingosine have been developed by using amino acids, ${ }^{7}$ carbohydrates $^{8}$ and other chiral building blocks ${ }^{9}$ as the precursors. However, most of the methods require multistep reactions (more than 10 steps) that resulted in low total yields (less than 10\%). As a continuing effort in seeking new glycolipids of immune stimulators, we thus explored a concise and efficient method for the synthesis of phytosphingosine. The starting material D-lyxose is an inexpensive sugar ${ }^{10}$ that possesses all the required chiral centers found in phytosphingosine. The retrosynthetic plan of phytosphingosine is depicted in Figure 1. Given these advantages, the amino group can be introduced by an $\mathrm{S}_{\mathrm{N}} 2$ replacement at the $\mathrm{C} 4$ position of lyxose, and the lipid chain can be introduced by Wittig olefination at the $\mathrm{C} 1$ position.

\footnotetext{
* Corresponding author. Tel.: +886 2 27898648; fax: +886 2 27835007; e-mail: cclin@chem.sinica.edu.tw
}

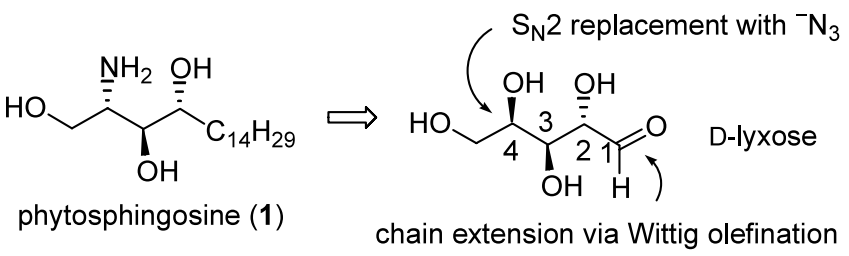

Figure 1. Retrosynthesis of phytosphingosine from D-lyxose.

The synthetic procedures, reagents and conditions are outlined in Scheme 1. Selective protection of 2,3 dihydroxyl groups of lyxose ${ }^{11}$ using 2,2-dimethoxypropane was followed by treatment with trityl chloride to give compound 2 (71\% yield). The subsequent condensation with a Wittig reagent $\mathrm{C}_{13} \mathrm{H}_{27} \mathrm{PPh}_{3} \mathrm{Br}$ (3) in the presence of lithium hexamethyldisilazide (LHMDS) afforded alkene 4 (93\% yield). ${ }^{12}$ The $E / Z$ ratio of 4 was determined to be $2: 1$ by ${ }^{1} \mathrm{H}$ NMR analysis. The double bond of $\mathbf{4}$ was reduced by catalytic hydrogenation to give $\mathbf{5}$ (91\% yield). ${ }^{13}$ Compound 5 was sequentially treated with trifluoromethanesulfonic anhydride $\left(\mathrm{Tf}_{2} \mathrm{O}\right)$ and tetramethylguanidinium azide (TMGA) ${ }^{14}$ to produce the azido compound $6(73 \% \text { yield })^{15}$ with inversion of the reacting carbon center. The acetal and trityl protecting groups in 6 were removed by an acid-catalyzed hydrolysis, and the azide group was reduced by Staudinger reaction ${ }^{16}$ using $\mathrm{Ph}_{3} \mathrm{P} / \mathrm{H}_{2} \mathrm{O}$ to give the desired product 1 as white solid. Thus, D-ribo- $C_{18}$-phytosphingosine $\mathbf{1}^{17}$ was synthesized from D-2,3- $O$-isopropylidene-D-lyxofuranose in $28 \%$ overall yield by this six-step procedure. This method was further simplified by using $\mathrm{Tf}_{2} \mathrm{O} /$ TMGA to convert alcohol 4 into azido compound 7 (84\% yield). ${ }^{18}$ After meticulous studies, we found a way 

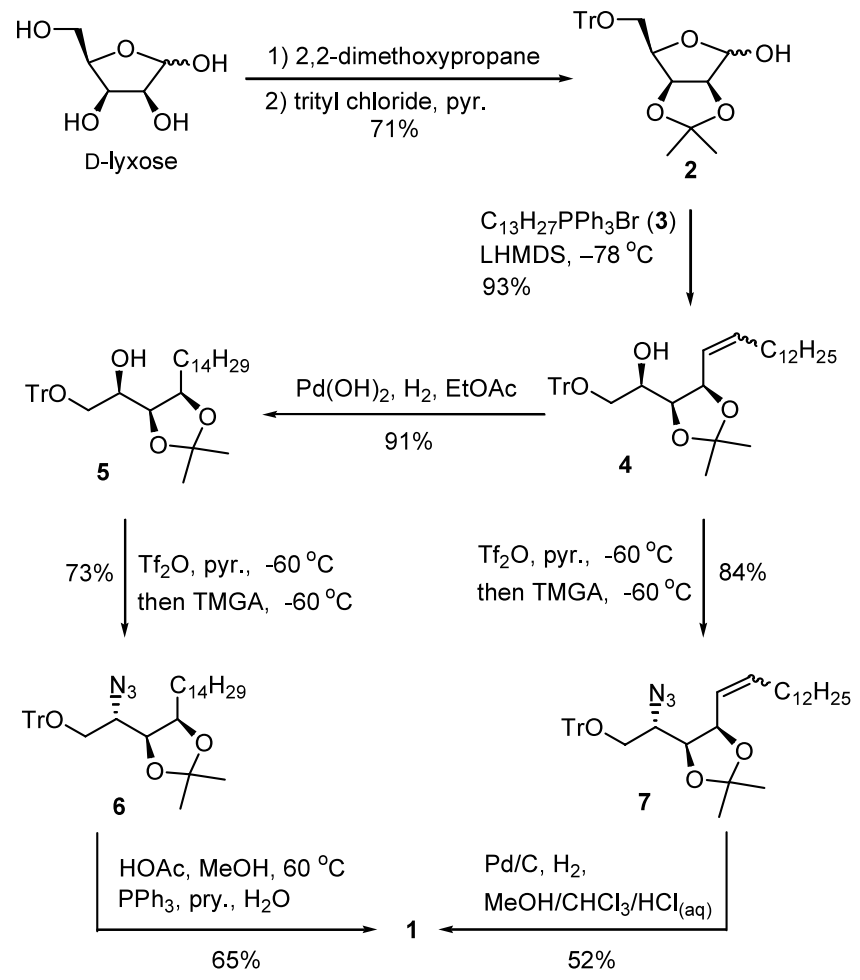

Scheme 1. Synthesis of phytosphingosine from D-lyxose.

to remove the acetal and trityl groups and simultaneously reduce the azide and double bond of 7 in a one-pot procedure. Thus, a solution of 7 in $\mathrm{CHCl}_{3} /$ $\mathrm{MeOH} / \mathrm{HCl}_{(\mathrm{aq})}(2 / 2 / 1)$ was treated with a catalytic amount of $\mathrm{Pd}(\mathrm{OH})_{2}$ under an atmosphere of hydrogen for $15 \mathrm{~h}$ at room temperature to give phytosphingosine 1 in $52 \%$ yield.

In conclusion, we have devised a very efficient method for the synthesis of phytosphingosine by using the inexpensive starting material $\mathrm{D}$-lyxose (2,3-O-isopropylidene-D-lyxofuranose is also the relatively inexpensive starting material). This method only requires six steps (from 2,3-O-isopropylidene-D-lyxofuranose) to give phytosphingosine in $28 \%$ overall yield. To our knowledge, this method is so far the shortest and relatively high-yielding route for a potential large-scale synthesis of phytosphingosine. By using different Wittig reagents, our method can also be modified to prepare a variety of sphingosine analogs.

\section{Acknowledgements}

The authors thank the Academia Sinica, National Science Council in Taiwan (NSC 91-3112-P-001-041-Y and NCS 91-2323-B-001-002), and National Taiwan University for their financial support.

\section{References}

1. (a) Kaufer, J. M.; Hakomori, S. In Handbook of Lipids Research: Sphingolipid Biochemistry; Kanfer, J. N.; Hakomori, S., Eds.; Plenum Press: New York, 1983; Vol. 3, pp. 1-150; (b) Kolter, T.; Sandhoff, K. Angew. Chem., Int. Ed. 1999, 38, 1532-1568.

2. (a) Chang, Y.-T.; Choi, J.; Ding, S.; PrieschI, E. E.; Baumruker, T.; Lee, J.-M.; Chung, S.-K.; Schultz, P. G. J. Am. Chem. Soc. 2002, 124, 1856-1857; (b) Turinsky, J.; Nagel, G. W. Biochem. Biophys. Res. Commun. 1992, 188, 358-364; (c) Dharmawardhane, S.; Rubinstein, B.; Stern, A. I. Plant Physiol. 1989, 89, 1345-1350; (d) Merrill, A. H., Jr.; Nimkar, S.; Menaldino, D.; Hannun, Y. A.; Loomis, C.; Bell, R. M.; Tyagi, J. D.; Lambeth, J. D.; Stevens, V. L.; Hunter, R.; Liotta, D. C. Biochemistry 1989, 28, 3138-3145; (e) Honda, M.; Ueda, Y.; Sugiyama, S.; Komori, T. Chem. Pharm. Bull. 1991, 39, 1385-1391; (f) Natori, T.; Morita, M.; Akimoto, K.; Koezuka, Y. Tetrahedron 1994, 50, 2771-2784.

3. (a) Kolter, T.; Sandhoff, K. Angew. Chem., Int. Ed. 1999, 38, 1532-1568; (b) Koskinen, P. M.; Koskinen, A. M. P. Synthesis 1998, 1075-1091; (c) Merrill, A. H., Jr.; Sweeley, C. C. In Biochemistry of Lipids, Lipoproteins and Membranes; Vance, D. E.; Vance, J. E., Eds.; Elsevier Science: Amsterdam, 1996; Chapter 12, pp. 309-339.

4. Natori, T.; Morita, M.; Akimoto, K.; Koezuka, Y.; Agelasphins, Y. Tetrahedron 1994, 50, 2771-2784.

5. Burdin, M.; Kronenberg, M. Curr. Opin. Immunol. 1999, 11, 326-331.

6. (a) Miyamoto, K.; Miyake, S.; Yamamura, T. Nature 2001, 413, 531-534; (b) Kawano, T.; Cui, J.; Koezuka, Y.; Youra, I.; Kaneko, Y.; Motoki, K.; Ueno, H.; Nakagawa, R.; Sato, H.; Kondo, E.; Koseki, H.; Taniguchi, M. Science 1997, 278, 1626-1629.

7. For recent examples using amino acid precursors, see: (a) Azuma, H.; Tamagaki, S.; Ogino, K. J. Org. Chem. 2000, 65, 3538-3541; (b) Takikawa, H.; Muto, S.-e.; Mori, K. Tetrahedron 1998, 54, 3141-3150; (c) Imashiro, R.; Sakurai, O.; Yamashita, T.; Horikawa, H. Tetrahedron 1998, 54, 10657-10670; (d) Yoda, H.; Oguchi, T.; Takabe, K. Tetrahedron: Asymmetry 1996, 7, 2113-2116; (e) Dondoni, A.; Fantin, G.; Fogagnolo, M.; Pedrini, P. J. Org. Chem. 1990, 55, 1439-1446; (f) Sugiyama, S.; Honda, M.; Komori, T. Liebigs Ann. Chem. 1990, 10691078.

8. For recent examples using sugar precursors, see: (a) Plettenburg, O.; Bodmer-Narkevitch, V.; Wong, C.-H. J. Org. Chem. 2002, 67, 4559-4564; (b) Luo, S.-Y.; Thopate, S. R.; Hsu, C.-Y.; Hung, S.-C. Tetrahedron Lett. 2002, 43, 4889-4892; (c) Graziani, A.; Passacantilli, P.; Piancatelli, G.; Tani, S. Tetrahedron: Asymmetry 2000, 11, 3921-3937; (d) Figueroa-Pérez, S.; Schmidt, R. R. Carbohydr. Res. 2000, 328, 95-102; (e) Murakami, T.; Taguchi, K. Tetrahedron 1999, 55, 989-1004; (f) Wee, A. G. H.; Tang, F. Tetrahedron Lett. 1996, 37, 6677-6680; (g) Li, Y.-L.; Mao, X.-H.; Wu, Y.-L. J. Chem. Soc., Perkin Trans. 1 1995, 1559-1563; (h) Matsumoto, K.; Ebata, T.; Matsushita, H. Carbohydr. Res. 1995, 279, 93-106.

9. For recent examples using various chiral precursors, see: (a) Nakamura, T.; Shiozaki, M. Tetrahedron 2001, 57, 9087-9092; (b) He, L.; Byun, H.-S.; Bittman, R. J. Org. Chem. 2000, 65, 7618-7626; (c) Martin, C.; Prünck, W.; 
Bortolussi, M.; Bloch, R. Tetrahedron: Asymmetry 2000, 11, 1585-1592.

10. D-Lyxose is not a usual and cheap sugar, but is also not a rare sugar.

11. Barbat, J.; Gelas, J.; Horton, D. Carbohydr. Res. 1991, 219, 115-121.

12. Harcken, C.; Martin, S. F. Org. Lett. 2001, 3, 3591-3593. Selective data for compound 4: The $E / Z$ ratio is $2: 1 .{ }^{1} \mathrm{H}$ NMR $\left(400 \mathrm{MHz}, \mathrm{CDCl}_{3}\right) \delta 0.88\left(\mathrm{t}, J=6.8 \mathrm{~Hz}, 3 \mathrm{H}_{Z}\right.$, $\left.3 \mathrm{H}_{E}\right), 1.25\left(\mathrm{br}, 20 \mathrm{H}_{Z}, 20 \mathrm{H}_{E}\right), 1.38\left(\mathrm{~s}, 3 \mathrm{H}_{Z}\right), 1.39\left(\mathrm{~s}, 3 \mathrm{H}_{E}\right)$, $1.49\left(\mathrm{~s}, 3 \mathrm{H}_{Z}, 3 \mathrm{H}_{E}\right), 1.74-1.84\left(\mathrm{~m}, 1 \mathrm{H}_{E}\right), 1.89-2.05(\mathrm{~m}$, $\left.1 \mathrm{H}_{E}, 2 \mathrm{H}_{Z}\right), 2.37\left(\mathrm{~d}, J=6.0 \mathrm{~Hz}, 1 \mathrm{H}_{E}, 1 \mathrm{H}_{Z}\right), 3.08-3.20(\mathrm{~m}$, $\left.2 \mathrm{H}_{E}, 1 \mathrm{H}_{Z}\right), 3.24\left(\mathrm{dd}, J=4.8,9.6 \mathrm{~Hz}, 1 \mathrm{H}_{Z}\right), 3.66-3.79(\mathrm{~m}$, $\left.1 \mathrm{H}_{E}, 1 \mathrm{H}_{Z}\right), 4.22\left(\mathrm{dd}, J=4.0,6.8 \mathrm{~Hz}, 1 \mathrm{H}_{E}\right), 4.26(\mathrm{dd}$, $\left.J=4.6,6.6 \mathrm{~Hz}, 1 \mathrm{H}_{Z}\right), 4.91\left(\mathrm{dd}, J=6.8,8.0 \mathrm{~Hz}, 1 \mathrm{H}_{E}\right)$, 5.47-5.61 (m, 2H $\left.\mathrm{H}_{E}\right), 7.18-7.32\left(\mathrm{~m}, 9 \mathrm{H}_{E}, 9 \mathrm{H}_{Z}\right), 7.40-7.50$ $\left(\mathrm{m}, 6 \mathrm{H}_{E}, 6 \mathrm{H}_{Z}\right)$.

13. Selective data for compound 5: $[\alpha]_{\mathrm{D}}^{26}=-3.7$ (c 1.0, $\left.\mathrm{CHCl}_{3}\right) ;{ }^{1} \mathrm{H} \mathrm{NMR}\left(400 \mathrm{MHz}, \mathrm{CDCl}_{3}\right) \delta 0.88(\mathrm{t}, J=6.0$ $\mathrm{Hz}, 3 \mathrm{H}), 1.26$ (br, 24H), 1.35 (s, 3H), 1.35-1.50 (m, 1H overlapped with $\mathrm{s}$ at $1.45 \mathrm{ppm}, 3 \mathrm{H}), 1.55-1.72(\mathrm{~m}, 1 \mathrm{H})$, $2.33(\mathrm{~d}, J=6.0 \mathrm{~Hz}, 1 \mathrm{H}), 3.13-3.25(\mathrm{~m}, 2 \mathrm{H}), 3.65-3.76(\mathrm{~m}$, $1 \mathrm{H}), 4.00-4.16(\mathrm{~m}, 2 \mathrm{H}), 7.15-7.35(\mathrm{~m} \mathrm{9H}), 7.35-7.50(\mathrm{~m}$, $6 \mathrm{H})$.

14. Tuch, A.; Saniére, M.; Merrer, Y. L.; Depezay, J.-C. Tetrahedron: Asymmetry 1996, 7, 897-906.
15. Selective data for compound 6: $[\alpha]_{\mathrm{D}}^{26}=9.0\left(c 0.4, \mathrm{CHCl}_{3}\right)$; ${ }^{1} \mathrm{H} \mathrm{NMR}\left(400 \mathrm{MHz}, \mathrm{CDCl}_{3}\right) \delta 0.91(\mathrm{t}, J=6.8 \mathrm{~Hz}, 3 \mathrm{H})$, 1.29 (br, $30 \mathrm{H}$ overlapped with two s at $1.25 \mathrm{ppm}, 3 \mathrm{H}$, $1.28 \mathrm{ppm}, 3 \mathrm{H}), 1.40-1.65(\mathrm{~m}, 2 \mathrm{H}), 3.88(\mathrm{dd}, J=7.6,10.0$ $\mathrm{Hz}, 1 \mathrm{H}), 3.51(\mathrm{ddd}, J=2.4,7.6,9.2 \mathrm{~Hz}, 1 \mathrm{H}), 3.56(\mathrm{dd}$, $J=2.4,9.6 \mathrm{~Hz}, 1 \mathrm{H}), 3.91(\mathrm{dd}, J=5.4,8.8 \mathrm{~Hz}, 1 \mathrm{H})$, 4.08-4.15 (m, 1H), 7.20-7.40 (m, 9H), 7.45-7.55 (m, 6H).

16. Kratzer, B.; Mayer, T. G.; Schmidt, R. R. Eur. J. Org. Chem. 1998, 291-298.

17. The data of D-ribo- $C_{18}$-phytosphingosine $\mathbf{1}$ was consisted with the given data of the following reference: Taguchi, K.; Murakami, T. Tetrahedron 1999, 55, 989-1004.

18. Selective data for compound 7: The $E / Z$ ratio is $2: 1{ }^{1} \mathrm{H}$ NMR (400 MHz, $\left.\mathrm{CDCl}_{3}\right) \delta 0.879\left(\mathrm{t}, J=6.8 \mathrm{~Hz}, 1 \mathrm{H}_{E}\right)$, $0.882\left(\mathrm{t}, J=6.8 \mathrm{~Hz}, 2 \mathrm{H}_{Z}\right), 1.26\left(\mathrm{br}, 20 \mathrm{H}_{E}, 20 \mathrm{H}_{Z}\right.$, overlapped with $2 \mathrm{~s}$ at $1.27 \mathrm{ppm}, 3 \mathrm{H}_{E}, 1.29 \mathrm{ppm}, 3 \mathrm{H}_{E}$ ), $1.34-1.43\left(\mathrm{~m}, 1 \mathrm{H}_{Z}\right.$ overlapped with s at $\left.1.39 \mathrm{ppm}, 3 \mathrm{H}_{Z}\right)$, $1.47\left(\mathrm{~s}, 3 \mathrm{H}_{Z}\right), 1.72-1.82\left(\mathrm{~m}, 1 \mathrm{H}_{Z}\right), 2.00-2.20\left(\mathrm{~m}, 2 \mathrm{H}_{E}\right)$, $3.17\left(\mathrm{dd}, J=5.2,11.2 \mathrm{~Hz}, 1 \mathrm{H}_{Z}\right), 3.32(\mathrm{dd}, J=8.8,10.4$ $\left.\mathrm{Hz}, 1 \mathrm{H}_{E}\right), 3.43-3.52\left(\mathrm{~m}, 2 \mathrm{H}_{E}, 1 \mathrm{H}_{Z}\right), 3.94(\mathrm{dd}, J=6.2,8.4$ $\left.\mathrm{Hz}, 1 \mathrm{H}_{E}\right), 4.51\left(\mathrm{dd}, J=6.2,8.4 \mathrm{~Hz}, 1 \mathrm{H}_{Z}\right), 4.78(\mathrm{dd}$, $\left.J=6.0,10.0 \mathrm{~Hz}, 1 \mathrm{H}_{Z}\right), 4.94\left(\mathrm{~m}, 1 \mathrm{H}_{E}, 1 \mathrm{H}_{Z}\right), 5.22-5.35(\mathrm{~m}$, $1 \mathrm{H}_{Z}$, overlapped with tdd at $5.31 \mathrm{ppm}, J=1.6,9.6,10.8$ $\left.\mathrm{Hz}, 1 \mathrm{H}_{E}\right), 5.41\left(\mathrm{td}, J=5.4,11.0 \mathrm{~Hz}, 2 \mathrm{H}_{Z}\right), 5.70$ (dtd, $\left.J=0.8,7.6,10.8 \mathrm{~Hz}, 1 \mathrm{H}_{E}\right), 7.20-7.35\left(\mathrm{~m}, 9 \mathrm{H}_{E}, 9 \mathrm{H}_{Z}\right)$, $7.38-7.52\left(\mathrm{~m}, 6 \mathrm{H}_{E}, 6 \mathrm{H}_{Z}\right)$. 